\title{
ALFVÉN WAVES AND TURBULENCE IN THE SOLAR ATMOSPHERE AND SOLAR WIND
}

\author{
ANDREa Verdini \\ Dipartimento di Astronomia e Scienza dello Spazio, Universitá degli Studi di Firenze, Firenze, Italy \\ AND \\ Marco Velli ${ }^{1}$ \\ Jet Propulsion Laboratory, California Institute of Technology, Pasadena, CA \\ Received 2006 August 5; accepted 2006 November 3
}

\begin{abstract}
We solve the problem of propagation and dissipation of Alfvénic turbulence in a model solar atmosphere consisting of a static photosphere and chromosphere, transition region, and open corona and solar wind using a phenomenological model for the turbulent dissipation based on wave reflection. We show that most of the dissipation for a given wavefrequency spectrum occurs in the lower corona, and the overall rms amplitude of the fluctuations evolves in a way consistent with observations. The frequency spectrum for a Kolmogorov-like slope is not found to change dramatically from the photosphere to the solar wind; however, it does preserve signatures of transmission throughout the lower atmospheric layers, namely, oscillations in the spectrum at high frequencies reminiscent of the resonances found in the linear case. These may disappear once more realistic couplings for the nonlinear terms are introduced or if timedependent variability of the lower atmospheric layer is introduced.
\end{abstract}

Subject headings: MHD — solar wind — turbulence — waves

Online material: color figures

\section{INTRODUCTION}

In situ measurement of magnetic and velocity field fluctuations from Helios and Ulysses have revealed a broad developed spectrum for frequencies ranging from $10^{-4}$ to $10^{-2} \mathrm{~Hz}$. Typically, a strong correlation between magnetic field and velocity fluctuations in this distance range persists (Mangeney et al. 1991), corresponding to an outwardly propagating spectrum. It is well-known that nonlinear terms couple Alfvén waves propagating in opposite directions. Also, the basic nonlinearity in homogeneous MHD in the presence of a majority of one type of waves forces the evolution with time to increase the dominance, preferentially dissipating the minority component in a process called dynamical alignment (Dobrowolny et al. 1980), which is not observed in the solar wind. Therefore, the presence of a well-developed spectrum together with a preferred direction of propagation has remained a mystery. The question that naturally arises therefore concerns the drivers for the continuing and anomalous (compared to homogeneous MHD predictions) nonlinear cascade in this outwardly dominant case. Among the possible drivers of a nonlinear cascade in the solar atmosphere are compressible effects, which couple Alfvén waves with slow and fast modes, or couplings due to the strong gradients in the atmosphere. Among the first are phenomena such as parametric decay (Pruneti \& Velli 1997; Del Zanna et al. 2001) and wave steepening (Suzuki $\&$ Inutsuka 2005). Gradients transverse to the mean magnetic field directions lead to phase mixing, i.e., development of small scales in directions perpendicular to that of propagation. Finally, the gradients due to stratification cause wave reflection, which naturally produces waves propagating in the opposite direction required for the classical incompressible cascade, as first suggested by Velli et al. (1989). Disentangling the role of all of these processes at once would require fully three-dimensional

\footnotetext{
${ }^{1}$ On leave of absence from the Dipartimento di Astronomia e Scienza dello Spazio, Universitá degli Studi di Firenze, Firenze, Italy.
}

calculations in a realistic atmosphere model, a feat beyond present numerical capabilities. We therefore focus here on the role of wave reflection, which has been extensively studied in the linear case (Heinemann \& Olbert [1980], Leroy [1980], and Hollweg [1978] were among the first), while less so in the nonlinear one (Matthaeus et al. 1983, 1994).

Some constraints on the frequency spectrum and the energies for the outward- and inward-propagating components are derived from the observations. The Alfvénic fluctuation power spectrum in the fast solar wind evolves with distance $(R)$, not self-similarly, with a power-law dependence on $\omega$ with slope -1 and $-5 / 3$ at low and high frequencies, respectively. The two intervals are separated by a critical frequency $\left(\omega_{*}\right)$, which depends on $R$. In identifying the fluctuations with Alfven waves, it is useful to adopt the Elsässer variables $\boldsymbol{z}^{ \pm}=\boldsymbol{u} \mp \operatorname{sgn}\left(B_{0}\right) \boldsymbol{b} /(4 \pi \rho)^{1 / 2}$ (corresponding, respectively, to outward- and inward-propagating Alfvén waves if the mean magnetic field $B_{0}$ is pointing outward from the Sun). The energy per unit mass residing in the outward- and inwardpropagating modes $\left(E^{ \pm}=\left|z^{ \pm}\right|^{2}\right.$, respectively) decreases with distance for both, and for $R<2.5 \mathrm{AU}, E^{+} \propto R^{-1.48}$, and $R^{-} \propto$ $E^{-0.42}$ (Bavassano et al. 2000b). The normalized cross helicity $\sigma_{c}=\left(E^{+}-E^{-}\right) /\left(E^{+}+E^{-}\right)$, which accounts for the imbalance between the outward and inward component, also evolves with distance, and it is approximately equal to 1 in the inner solar wind. It decreases for $R>0.4 \mathrm{AU}$ and oscillates around $\approx 0.4$ for $R>2.5 \mathrm{AU}$ (Bavassano et al. 2000a).

It must be recalled that the observed features cannot be explained either by linear propagation theory (including reflection) or by MHD turbulence separately. A linear analysis applied to the solar wind case shows that low-frequency waves $\left(\omega<10^{-5} \mathrm{~Hz}\right)$ experience the strongest reflection in the photosphere, chromosphere, and corona (Hollweg 1978, 1981; Similon \& Zargham 1992). Their flux at the transition region is greatly reduced (even if considerable power is transmitted to the corona), and in the outer (supersonic) solar wind the radial dependence of $\sigma_{c}$ deduced from observations is similar to the radial dependence one 
finds at higher frequencies in the linear regime (Velli et al. 1991). On the other hand, the high-frequency waves $\left(10^{-4} \mathrm{~Hz}<\omega<\right.$ $10^{-2} \mathrm{~Hz}$ ) are almost completely transmitted (even if in the photosphere and chromosphere their reflection is relatively high), both the $E^{+}$and $E^{-}$energies decrease with distance faster than the scaling law deduced from observation, and finally, $\sigma_{c} \approx 1$ in the outer solar wind (Velli et al. 1991). The dynamics of a welldeveloped turbulent state in the expanding solar wind has been studied as well, and ordering of the characteristic timescale, which should effectively favor the development of a turbulent cascade in planes perpendicular to the direction of wave propagation (along the magnetic field), has been found (Zank et al. 1996; Matthaeus et al. 1998, 1999; Dmitruk et al. 2001a, 2002; Dmitruk \& Matthaeus 2003; Oughton et al. 2001, 2004). Numerical models capable of reproducing the observed $\sigma_{c}$-profiles in the supersonic part of the solar wind (Zhou \& Matthaeus 1989, 1990) or the spectral evolution (Tu et al. 1984; Tu 1988; Velli et al. 1989) necessarily use ad hoc assumptions and simplifications, and even if considerable advances have been made, a complete understanding of the solar turbulent spectrum and the solar wind acceleration (Li et al. 1999; Habbal et al. 1995) has not been achieved.

Here we investigate the combined effect of wave reflection and turbulent dissipation in order to understand the relative importance of linear and nonlinear effects on the overall evolution of the fluctuation amplitudes. Comparisons of the numerical results with some observations give some constraints on the fields at the photospheric and coronal level for which data are still missing, with implications for numerical models of solar wind acceleration. In the context of a reflection-driven turbulent cascade process, another interesting issue concerns the evolution of the turbulent spectrum. If one supposes that the Alfvén waves are injected at the photospheric base at a well-defined frequency or with a given correlation time, one would expect to find a signature of this characteristic timescale in the observed spectrum at $1 \mathrm{AU}$ (or in other words, one can ask if discrete modes and turbulence can coexist; Dmitruk et al. 2004). No injection frequency is observed in the solar wind spectrum, so one can ask if both the turbulent evolution and the frequencydependent transmission properties of the solar atmosphere and wind can efficiently smooth this supposed strong forcing signature.

We numerically integrate the equations for the velocity and magnetic field fluctuations (written in terms of the Elsässer fields) for a stationary model atmosphere with a supraspherically expanding wind, from the photosphere to $1 \mathrm{AU}$ for a set of frequencies chosen in the range $10^{-6} \mathrm{~Hz}<\omega<10^{-2} \mathrm{~Hz}$. Each wave is identified via its frequency, while a phenomenological nonlinear term is added to the equations in order to account for both turbulent dissipation and frequency coupling.

\section{THE MODEL}

The equations describing the propagation of Alfvén waves in an inhomogeneous stationary medium can be derived from the MHD equations under the assumption of incompressible adiabatic transverse fluctuations. The velocity $(\boldsymbol{u})$ and magnetic field fluctuations $(\boldsymbol{b})$ are combined to form the Elsässer variables, which describe Alfvén waves propagating outward $\left(z^{+}\right)$or inward $\left(z^{-}\right)$. In terms of these variables, the equation for the two fields reads

$$
\begin{aligned}
\frac{\partial \boldsymbol{z}^{ \pm}}{\partial t}+ & {\left[\left(\boldsymbol{U} \pm \boldsymbol{V}_{\mathrm{A}}\right) \cdot \nabla\right] \boldsymbol{z}^{ \pm}+\left(\boldsymbol{z}^{\mp} \cdot \nabla\right)\left(\boldsymbol{U} \mp \boldsymbol{V}_{\mathrm{A}}\right) } \\
& \pm \frac{1}{2}\left(\boldsymbol{z}^{\mp}-\boldsymbol{z}^{ \pm}\right)\left[\nabla \cdot \boldsymbol{V}_{\mathrm{A}} \mp \frac{1}{2}(\nabla \cdot \boldsymbol{U})\right]=-\left(\boldsymbol{z}^{\mp} \cdot \nabla\right) \boldsymbol{z}^{ \pm}
\end{aligned}
$$

where $\boldsymbol{U}$ is the mean wind speed, the Alfvén speed is $\boldsymbol{V}_{\mathrm{A}}=$ $\boldsymbol{B}_{0} /(4 \pi \rho)^{1 / 2}$, and colinearity between the magnetic and gravitational fields is assumed. On the right-hand side we have grouped the nonlinear terms (except the total pressure, which in the limit of incompressible fluctuations can also be written as the product of $z^{+}, z^{-}$, and their gradients). In the linear part of equation (1) we can recognize a propagation term (second term) and two terms accounting for reflection due to the variation of the properties of the medium, one isotropic (fourth term) and the other (third term) involving variations along the fluctuations' polarization.

The chromosphere and the photosphere are modeled as a static layer, $2400 \mathrm{~km}$ thick, with the magnetic field organized in a flux tube in supraspherical geometry with constant temperature. The density varies almost exponentially, and the magnetic field varies according to the flux tube expansion $(A)$ in order to reproduce the properties of a coronal hole in the quiet Sun (Hollweg et al. 1982). Across the transition region, the density falls off by 2 orders of magnitude, and the wind passes from a speed of 0 to $8 \mathrm{~km} \mathrm{~s}^{-1}$, while the magnetic field strength is continuous (about $10 \mathrm{G}$ ). The corona also expands supraspherically, and its temperature profile is chosen to fit observations (see Fig. 1); it starts at $8 \times 10^{5} \mathrm{~K}$ at the coronal base, peaks at about $3 \times 10^{6} \mathrm{~K}$ at $3 R_{\odot}$, and then falls off with distance as $r^{-0.7}$ (Casalbuoni et al. 1999). The wind speed profile follows from the wind equations with a given temperature and flux tube expansion of the form $A(r)=f(r) r^{2}$, where $f$ is a function that has a maximum close to the coronal base and tends to a finite value at large distances (see Kopp \& Holzer 1976; Munro \& Jackson 1977). The same functional form is chosen for the expansion in the static part of the atmosphere, but different parameters are selected in order to obtain realistic values for the magnetic field and its continuous variation at the transition region. In the photosphere and chromosphere, the profile for Alfvén speed is obtained from magnetic flux conservation, $B=B_{0} A_{0} / A(r)$, and the density profile imposed.

Following Dmitruk et al. (2001b) we choose the following model for the nonlinear terms in equation (1),

$$
\mathrm{NL}_{j}^{ \pm}=z^{ \pm}\left(\omega_{j}\right) \frac{\left|Z^{\mp}\right|}{L(r)},
$$

where $L$ represents an integral turbulent dissipation length and $\left|Z^{\mp}\right|$ stands for the total amplitude of the Elsässer field integrated over the whole spectrum $(\Omega)$ at the point $r$; hence, $\left|Z^{\mp}\right|=$ $\left\{\int_{\Omega}\left[\left|z^{\mp}(\omega)\right|^{2} / \omega\right] d \omega\right\}^{1 / 2}$. This choice overestimates the transfer rate between high-frequency modes, for which the Alfvén effect is important (Shebalin et al. 1983). In reality, the predominant interaction, as seen below, concerns the lowest frequency reflected mode and the full outward-propagating spectrum, for which the resonance effects are not important.

The energy distribution among the modes influences the dissipation rate of all the waves coupled. In particular, at a fixed total rms energy, dissipation is reduced if the energy of the higher frequency waves is comparable to the lower frequency ones (flatter spectra) with respect to the case in which most of the energy is contained in the low-frequency modes (steeper spectra; Verdini et al. 2005). Equation (1) can be simplified by including the systematic variation of the Elsässer amplitude in a new normalized variable $z_{N}^{ \pm}=z_{O}^{ \pm}\left(M_{\mathrm{A}} \pm 1\right) /\left(M_{\mathrm{A}}\right)^{1 / 2}$, which reduces to $z_{N}^{ \pm}=$ $\pm z_{O}^{ \pm} \rho^{1 / 4}$ in the limit of small Alfvénic Mach number $M_{\mathrm{A}}=$ $U / V_{\mathrm{A}} \rightarrow 0$ (see Heinemann \& Olbert 1980). After Fourier transforming the linear equations in time and adding the phenomenological nonlinear term, one obtains

$$
\left(U \pm V_{\mathrm{A}}\right) \boldsymbol{z}_{N}^{ \pm \prime}-i \omega \boldsymbol{z}_{N}^{ \pm}-\frac{1}{2}\left(U \pm V_{\mathrm{A}}\right) \frac{V_{\mathrm{A}}^{\prime}}{V_{\mathrm{A}}} \boldsymbol{z}_{N}^{\mp}=-\frac{\left|Z_{O}^{\mp}\right|}{L} \boldsymbol{z}_{N}^{ \pm},
$$



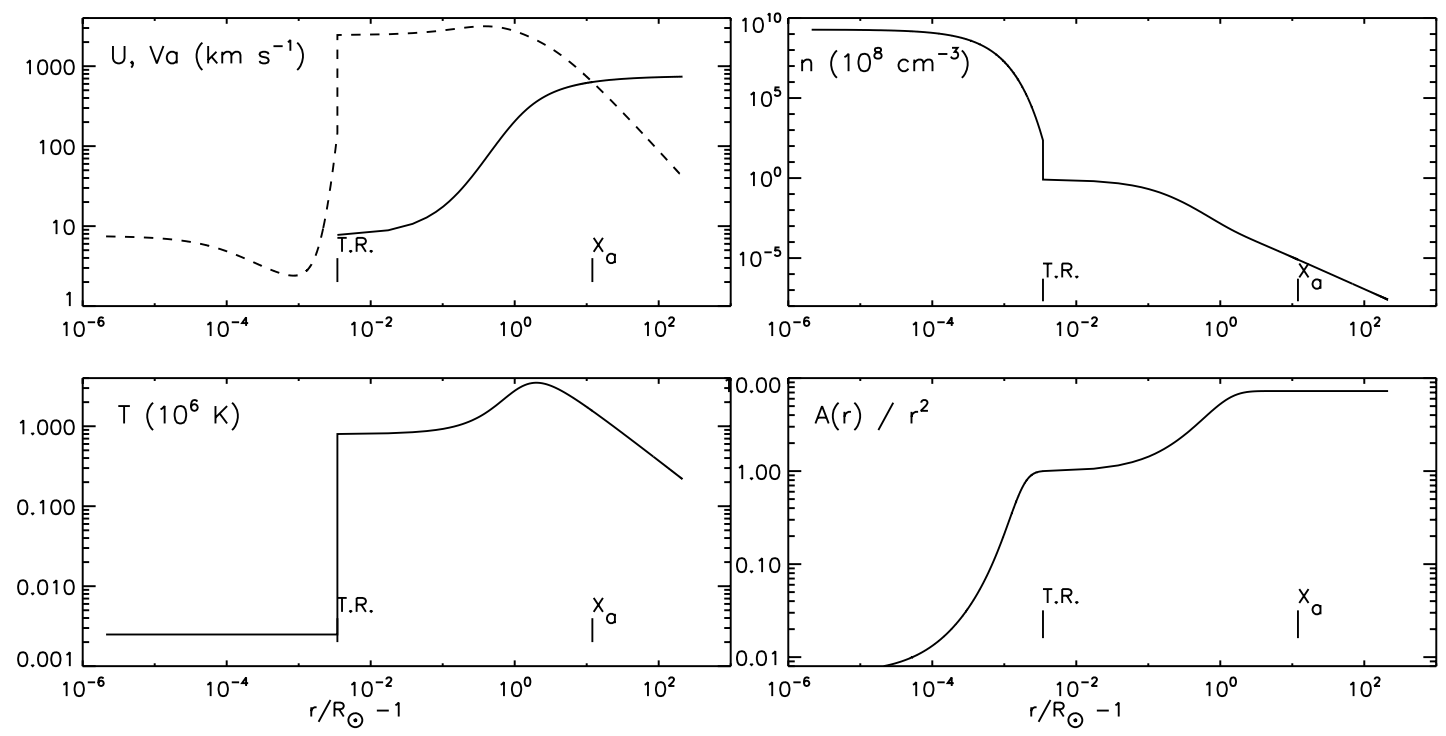

FIG. 1.-From top left, clockwise: Wind speed (solid line) and Alfvén speed (dotted line), numerical density, temperature, and expansion factor as functions of heliocentric distance for the modeled atmosphere.

where the prime indicates a derivative with respect to $r$. The numerically integrated equations are

$$
z_{N}^{ \pm^{\prime}}-i \frac{\omega}{U \pm V_{\mathrm{A}}} z_{N}^{ \pm}-\frac{1}{2} \frac{V_{\mathrm{A}}^{\prime}}{V_{\mathrm{A}}} z_{N}^{\mp}=-\frac{\left|Z_{O}^{\mp}\right|}{\left(U \pm V_{\mathrm{A}}\right) L} z_{N}^{ \pm}
$$

for the corona, while for the photosphere and the chromosphere one gets

$$
z_{N}^{ \pm^{\prime}} \mp i \frac{\omega}{V_{\mathrm{A}}} z_{N}^{ \pm}+\frac{1}{2} \frac{V_{\mathrm{A}}^{\prime}}{V_{\mathrm{A}}} z_{N}^{\mp}=\mp \frac{\left|Z_{O}^{\mp}\right|}{V_{\mathrm{A}} L} z_{N}^{ \pm} .
$$

The second, third, and last coefficients in equations (4) and (5) represent the propagation $(\mathrm{P})$, reflection $(\mathrm{R})$, and nonlinear dissipation (NL) coefficients, respectively (inverse of parallel wavelength, reflection scale height, and nonlinear length scale). The dissipative feature of the nonlinear terms can be shown by multiplying the above equation (3) in its old variables form by the complex conjugate $z^{ \pm *}$ to obtain the evolution equations for the Elsässer energies at a given frequency $E^{ \pm} \equiv \frac{1}{2}\left|z^{ \pm}(\omega)\right|^{2}$. On the right-hand side, one gets $-\left|z^{ \pm}\right|^{2}\left|\boldsymbol{Z}^{\mp}\right| / L$, which is independent of the phase difference between the two fields and involves the total amplitude of the fluctuations (the same term appears in the equation for a static atmosphere). In the presence of a wind, energy flux as a conserved quantity is replaced for linearly propagating waves by the total wave action flux, which can be written as the difference between an outgoing and ingoing flux,

$$
\begin{aligned}
S^{*}= & S^{+}-S^{-}=\frac{1}{4} \rho U A \\
& \times\left[\frac{U+V_{\mathrm{A}}}{U V_{\mathrm{A}}}\left(\boldsymbol{U}+\boldsymbol{V}_{\mathrm{A}}\right)\left|z^{+}\right|^{2}-\frac{U-V_{\mathrm{A}}}{U V_{\mathrm{A}}}\left(\boldsymbol{U}-\boldsymbol{V}_{\mathrm{A}}\right)\left|z^{-}\right|^{2}\right],
\end{aligned}
$$

where the superscripted plus and minus refer to the outward and inward direction, respectively, and $S$ is the wave action.

The inward wave action density vanishes at the Alfvén critical point $\left(X_{\mathrm{A}} \approx 13 R_{\odot}\right.$, where the Alfvén speed equals the wind speed), so one can write $S^{*}=S_{0}^{+}-S_{0}^{-}=S_{c}^{+}$, where the index $c$ stands for the critical point, while the index 0 refers to the base of the layer. The amplitude and phase of the outward-propagating Elsässer field $\left(z^{+}\right)$at $X_{\mathrm{A}}$ define the natural boundary conditions, since the critical point is a regular singular point for the incoming wave equation, because the phase velocity of the mode vanishes there; total wave action density is imposed, and the amplitude and phase of $z^{-}$can be derived by demanding the regularity of the solutions at $X_{\mathrm{A}}$. However, boundary conditions are chosen to ensure an amplitude of the rms velocity field fluctuations (i.e., summed over the whole spectrum) of $\approx 40 \mathrm{~km} \mathrm{~s}^{-1}$ at $1 R_{\odot}$, as constrained by observations (Banerjee et al. 1998), with an assigned spectral distribution; this requires some trial and error, since nonlinearity does not allow rescaling of the photospheric amplitude by simply rescaling values at the critical point $X_{\mathrm{A}}$. The shape of the photospheric spectrum is imposed approximately thanks to the quasi-linear properties of the waves in the photospherechromosphere layer (small wave amplitudes) and the fact that transmission and nonlinearity yield frequency-independent evolution in the low corona, as shown in $\S 3$. Given a slope $p$ at the Alfvénic critical point, the transmission coefficient of the static layer $T(\omega)$ (see Krogulec \& Musielak 1998 for discussion on it),

$$
T(\omega)=\frac{S_{c}^{+}}{S_{0}^{+}}=\frac{\rho_{c} V_{\mathrm{Ac}}}{\rho_{0} V_{\mathrm{A} 0}} \frac{\left|z_{c}^{+}\right|^{2}}{\left|z_{0}^{+}\right|^{2}}=\frac{\left|z_{N c}^{+}\right|^{2}}{\left|z_{N 0}^{+}\right|^{2}},
$$

can therefore be used to correct the initial spectrum $\left|z^{+}(\omega)\right|=$ $\left|z^{+}\left(\omega_{0}\right)\right|\left(\omega / \omega_{0}\right)^{p}$ to the desired spectrum at the photosphere, imposing $\left|z^{+}(\omega)\right|=\left|z^{+}\left(\omega_{0}\right)\right|[T(\omega)]^{1 / 2}\left(\omega / \omega_{0}\right)^{p}$. In order to describe the spectrum, 32 modes are chosen in the range of frequency between $10^{-6}$ and $10^{-2} \mathrm{~Hz}$ with increasing resolution at higher frequencies.

The phenomenological turbulent length scale varies as $L(r)=$ $L_{0}[A(r)]^{1 / 2}$, where $L_{0}=34,000 \mathrm{~km}$ is imposed at the coronal base and corresponds to the average size of the supergranules. The waves are propagated from the Alfvénic critical point forward (to Earth orbit) and backward (to the base of the corona) by the integration of equation (4). The conservation of the energy flux across the transition region allows one to determine the Elsässer 

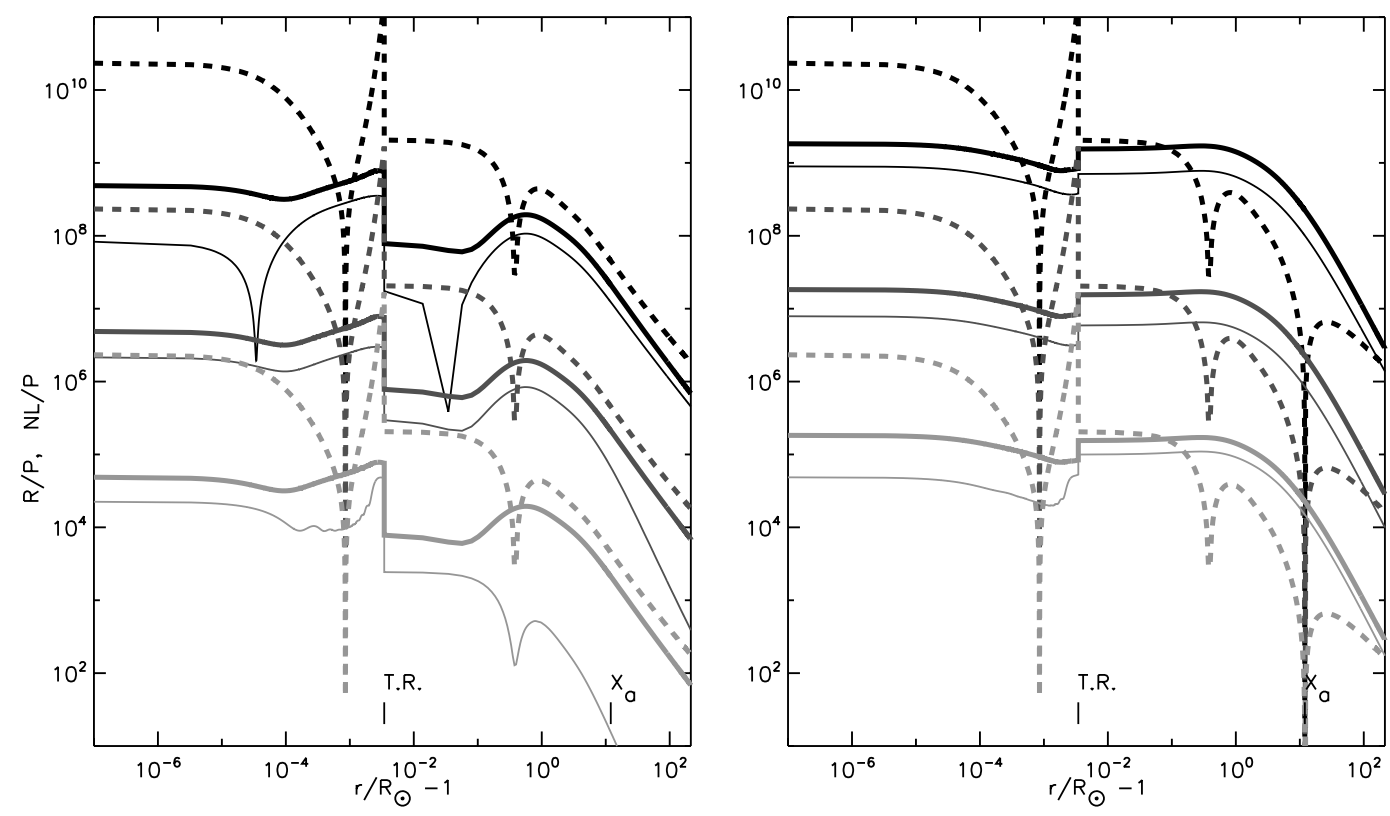

FIG. 2.-Comparison of the reflection ( $\mathrm{R}$; thick dashed line) and nonlinear coefficient (NL; thick solid line) normalized to the propagation coefficient (P) for the outgoing and ingoing wave at three different frequencies $\left(10^{-6}, 10^{-4}\right.$, and $10^{-2} \mathrm{~Hz}$; black, gray, and light gray lines, respectively). Also shown as a thin solid line is the contribution of each frequency wave to the nonlinear coefficient. The photospheric frequency spectrum is flat, and the boundary conditions at $X_{\mathrm{A}}$ are set to get an rms velocity field fluctuation at the coronal base $\delta u=40 \mathrm{~km} \mathrm{~s}^{-1}$. [See the electronic edition of the Journal for a color version of this figure.]

fields below the discontinuity, which are propagated back to the base of the photosphere using equation (5).

\section{RESULTS}

Following Velli (1993) we compare the characteristic length scales of equations (4) and (5) in the two layers. First consider the thick lines in Figure 2, which represent the reflection and nonlinear coefficients (solid and dashed lines, respectively) normalized to the propagation coefficient for $\omega=10^{-6}, 10^{-4}$, and $10^{-2} \mathrm{~Hz}$ (black, gray, and light gray lines, respectively) for a flat photospheric spectrum. Reflection has a maximum at the transition region, and it falls off by a factor of about 100 in the corona (because of the density drop). The zeros in the reflection coefficient appearing for both the $z^{+}$and the $z^{-}$depend on the fact that $V_{\mathrm{A}}^{\prime}=0$ (approximately in the corona), while the one located at $X_{\mathrm{A}}$ appears only for the backward-propagating waves, since the propagation coefficient becomes infinite there (see eq. [4]). For the outward-propagating wave (Fig. 2, left), reflection is generally much greater (a factor of 100) than dissipation in the photosphere-chromosphere and in the very low corona (below $\approx 1.2 R_{\odot}$ ). Further out, the nonlinear dissipation is smaller than the reflection but of the same order of magnitude. For the inward-propagating wave (Fig. 2, right), again reflection dominates in the photosphere-chromosphere (by a factor of 10), but in the corona the dissipative coefficient is comparable to or much greater than the reflection coefficient.

Hence, the relative dissipation of the linearly conserved quantities, as defined below in equation (8), has different features in the two layers. In Figure 3 we plot the total wave action density for the corona (main panel) and the total wave energy flux for the static layer (inset) normalized to their base value for all the frequencies that form the spectrum, i.e.,

$$
\begin{aligned}
\frac{S^{*}(r, \omega)}{S_{0}^{*}(\omega)}= & \frac{\left|z_{N}^{+}\right|^{2}-\left|z_{N}^{-}\right|^{2}}{\left|z_{N_{0}}^{+}\right|^{2}-\left|z_{N_{0}}^{-}\right|^{2}}=1-\frac{1}{2\left(\left|z_{N_{0}}^{+}\right|^{2}-\left|z_{N_{0}}^{-}\right|^{2}\right)} \\
& \times \int_{r_{0}}^{r} \frac{d r}{L V_{\mathrm{A}}}\left(\frac{\left|Z_{O}^{-}\right|}{1+M_{\mathrm{A}}}\left|z_{N}^{+}\right|^{2}+\frac{\left|Z_{O}^{+}\right|}{1-M_{\mathrm{A}}}\left|z_{N}^{-}\right|^{2}\right),
\end{aligned}
$$

with the normalization used to derive equation (3): the coefficients appearing in the integral are the nonlinear frequency-integrated coefficients discussed above.

In the upper chromosphere, the flux tube expansion is very rapid and the reflection is strong. Both the ingoing and outgoing waves contribute to the damping of the energy flux (comparableless than 1 order of magnitude difference - nonlinear coefficient and wave amplitudes), and the relative dissipation is very high. Low-frequency modes (Fig. 3, inset [lower plots]) are the most damped (the most reflected), while high-frequency modes (inset [top plot]) are the least damped. In fact, inspection of equation (8) reveals that the relative dissipation is quadratic in the frequencydependent wave amplitudes $\left(\left|z_{N}^{ \pm}\right|^{2}\right)$, which in turn increase with decreasing frequency because of the different reflection rate. In the corona, beyond $2 R_{\odot}$ the dissipation coefficient for the outgoing waves is weaker, and their amplitudes grow; reflection is

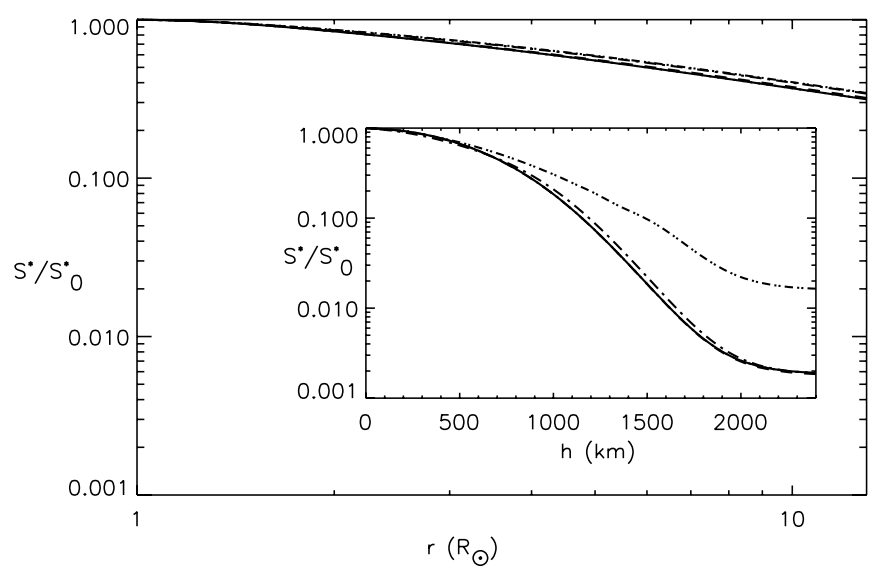

FIG. 3.-Normalized wave action density for the corona as a function of distance for five frequencies $\left(10^{-6} \mathrm{~Hz}\right.$, solid line; $10^{-5} \mathrm{~Hz}$, dotted line; $10^{-4} \mathrm{~Hz}$, dashed line $; 10^{-3} \mathrm{~Hz}$, dot-dashed line; $10^{-2} \mathrm{~Hz}$, double-dot-dashed line). The wave energy flux for the photosphere-chromosphere is plotted in the inset with the same line coding. 


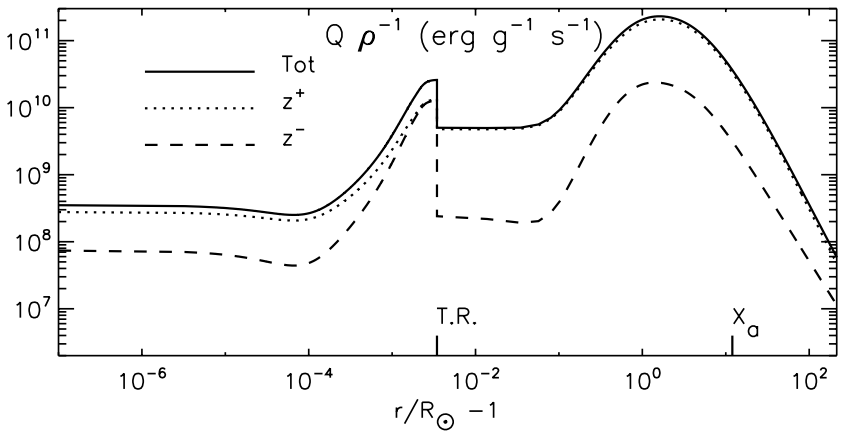

FIG. 4.- Heating rate per unit mass integrated over the whole spectrum as a function of heliocentric distance. The contributions of the ingoing (dashed line) and outgoing (dotted line) heating rates are also shown. The transition region (T.R.) and the Alfvénic critical point $\left(X_{\mathrm{A}} \approx 13 R_{\odot}\right)$ are indicated on the $x$-axis.

weaker as well, and an imbalance between outgoing and ingoing fluxes holds. Only the former contribute to the wave action density dissipation, since now the dominant quadratic dependence in equation (8) comes from the outgoing mode (see, e.g., the approximate conservation form used by Cranmer \& van Ballegooijen 2005). Note that for all frequencies the wave action density decreases at approximately the same rate. It turns out that the amplitude evolution is driven mainly by the nonlinear, frequency-independent term in the corona and by the reflection, frequency-dependent term in the photosphere-chromosphere, a feature we find again by studying the power spectrum evolution. In comparison, the heating rate per unit mass, an absolute measure of energy dissipation, integrated over the spectrum,

$$
\frac{Q}{\rho}=\frac{Q^{+}}{\rho}+\frac{Q^{-}}{\rho}=\frac{\left|Z^{+}\right|^{2}\left|Z^{-}\right|+\left|Z^{-}\right|^{2}\left|Z^{+}\right|}{L(r)},
$$

is generally higher in the corona than in the photospherechromosphere, as shown in Figure 4. In the latter layer, both the ingoing and outgoing wave contribute to the total amount of heating rate, while in the former most of the dissipation comes from the outgoing mode. The absolute dissipation is quadratic in the frequency-integrated wave amplitudes, and in the corona outgoing waves are allowed to grow almost undamped (low relative dissipation), but the existence of a small seed of ingoing waves ensures a large absolute dissipation. This is not true in the photospherechromosphere, before the rapid expansion of the flux tube, where the wave amplitude is small and there is a small imbalance between outgoing and ingoing propagating wave amplitudes.

The effect of a different slope of the initial spectrum can be understood by analyzing the contribution of each frequency to the nonlinear coefficient, plotted in thin lines in Figure 2. Starting with a flat photospheric frequency spectrum results in an approximately equal contribution to the total nonlinear term in the whole atmosphere, except for the outer corona, where the nonlinear coefficient for the outward-propagating wave is made up of essentially backward-propagating waves at low frequencies. Note also that the frequency-decomposed nonlinear coefficient is approximately the same for outgoing and ingoing propagating waves in the photosphere-chromosphere, since reflection is high enough compared to dissipation. It follows that if a Kolmogorovlike photospheric spectrum $\left(E / \omega \propto \omega^{-5 / 3}\right)$ is imposed, the nonlinear term is mainly made up of low-frequency waves for both counterpropagating waves in both the layers. This can be seen in Figure 5 by comparing the thick and thin solid lines; for $\omega \gtrsim 10^{-4} \mathrm{~Hz}$ the contribution to the nonlinear coefficient is generally less then $10 \%$. An exception is found below $2 R_{\odot}$ for the outgoing mode, since reflection is high even for intermediatefrequency waves (see Fig. 7 for the photospheric layer). Note that a dip in the (frequency-integrated) nonlinear coefficient for the outgoing mode appears below the location of vanishing reflection in both the photosphere and low corona, since the energy resides mainly in the low-frequency mode.

This separate behavior in the two layers has strong consequences on spectral evolution. In Figure 6 the (compensated) total power in the fluctuations is plotted for different heliocentric distances. An almost Kolmogorov-like spectrum is imposed at the base of the photosphere with the procedure described at the end of $\S 2$. At very low frequencies the spectrum practically does not evolve

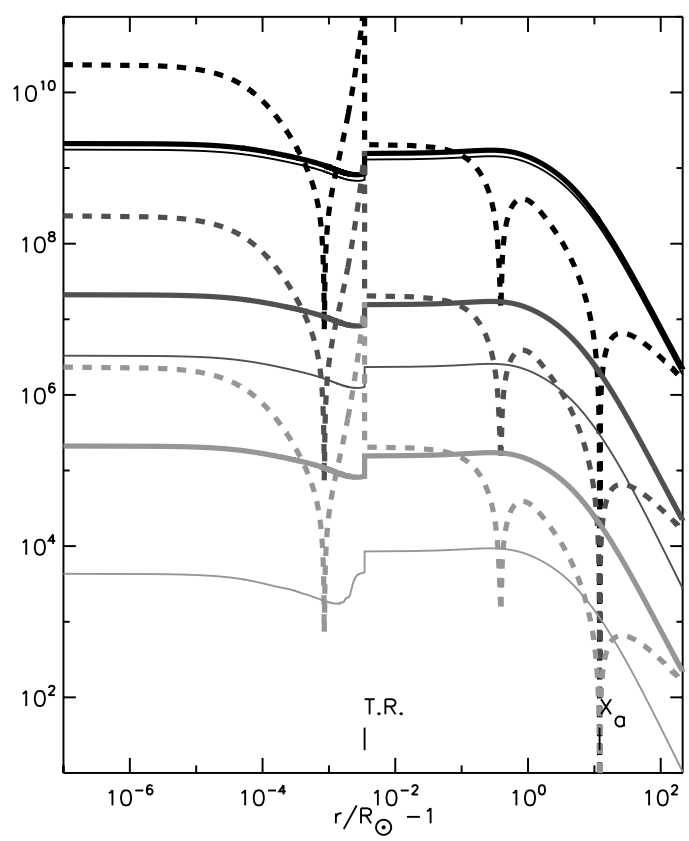




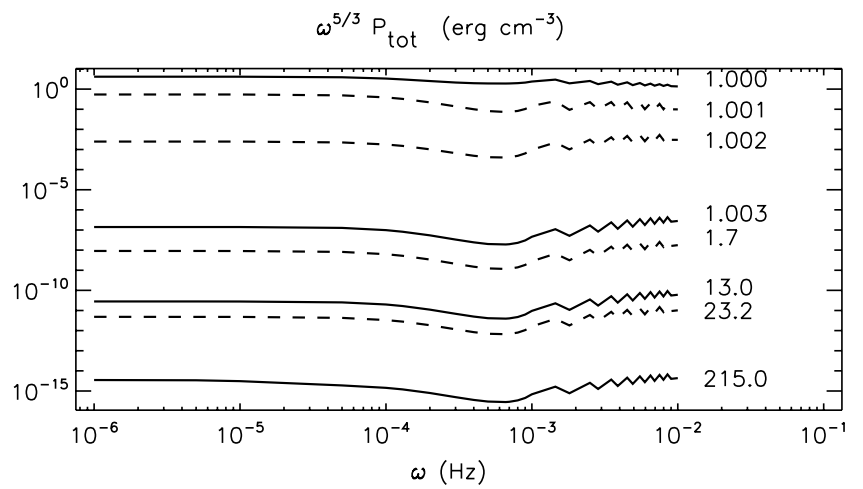

FIG. 6.-Compensated power spectrum as a function of heliocentric distance for a photospheric Kolmogorov-like initial spectrum. Each line is labeled with the corresponding heliocentric distance in units of $R_{\odot}$. From top to bottom, solid lines indicate the photospheric base, the transition region, $X_{\mathrm{A}}$, and $1 \mathrm{AU}$.

over the whole domain, while there is a tendency to steepen at low to intermediate frequencies $\left(10^{-5} \mathrm{~Hz} \lesssim \omega \lesssim 10^{-3} \mathrm{~Hz}\right)$. The behavior at high frequencies is quite complicated. Some irregularities appear very close to the base of the photosphere, and the overall tendency is that of flattening. Note, however, that most of the changes in the shape occur in the photosphere-chromosphere, where the waves display a strong frequency-dependent behavior. This makes the spectral evolution very similar to the linear case below the transition region (except the energy level of the spectrum), and the appearance of the irregularities can be interpreted by means of linear analysis. Accordingly, in Figure 7 we plot the transmission coefficient, defined in equation (7), as a function of frequency for the photosphere-chromosphere. Note that the transmission is constant at low frequency, decreases at intermediate frequencies, and increases again at high frequencies, where several transmission peaks appear; basically all spectral evolution is qualitatively reproduced. The peaks originate from the discontinuity in the reflection scale height at the transition region (Velli 1993). In fact, the amplitude of the reflected waves shows some nodes inside the domain, and when their location coincides with the base of the photosphere the transmission is enhanced (a condition that depends on the frequency of the waves; see Hollweg 1978). When nonlinearities are introduced, the location of the nodes depends also on the wave amplitude imposed at $X_{\mathrm{A}}$ (see Verdini et al. 2005), and similarly, if these nodes are located near the base of the photosphere, irregularities in the spectrum appear.

The slope of the spectrum imposed at the photosphere has negligible effects on the total power spectral evolution; however, it changes the amount of energy residing in the ingoing and outgoing modes (or in the kinetic or magnetic fluctuations) at large

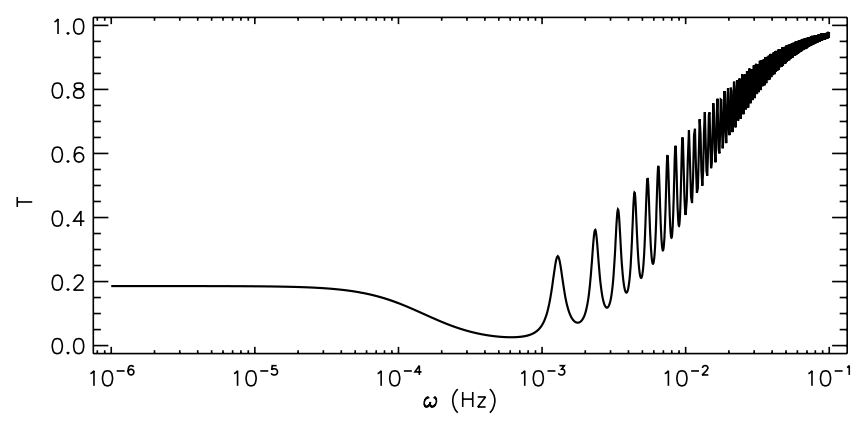

FIG. 7.-Transmission coefficient for the photosphere-chromosphere as a function of frequency.

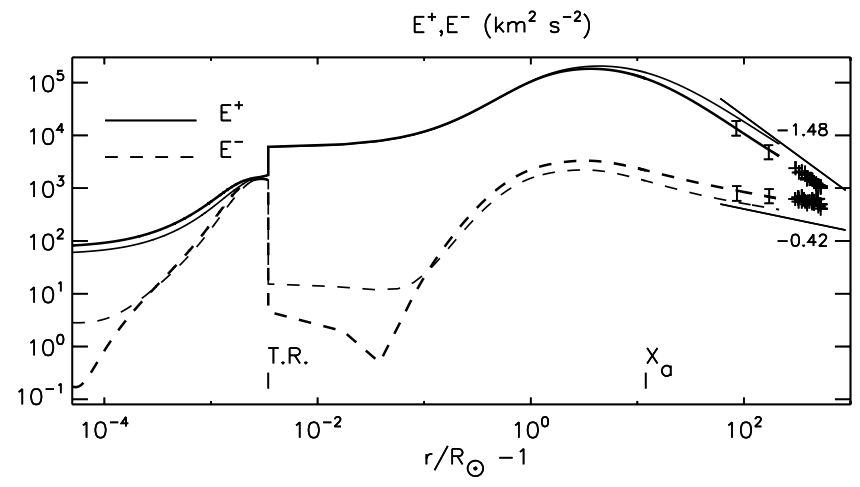

FIG. 8.-Frequency-integrated Elsässer energies as a function of heliocentric distance for a Kolmogorov and a flat photospheric spectrum with $\delta u=40 \mathrm{~km} \mathrm{~s}^{-1}$ at the coronal base (thick and thin lines, respectively). Symbols indicate observational constraints (see text for explanation).

distances, and some constraints on the slope can be obtained using the available observational data. In Figure 8 the Elsässer energies $E^{ \pm}$integrated over the frequency spectrum are plotted (solid and dashed lines, respectively) along with the Ulysses and Helios data (Bavassano et al. 2000b) for a Kolmogorov (thick lines) and a flat (thin lines) initial slope with $\delta u=40 \mathrm{~km} \mathrm{~s}^{-1}$ at the coronal base. Both the data and the expected slopes are reproduced by the Kolmogorov-like photospheric spectrum, while the flat one has an outgoing energy that is too high and an ingoing energy that is too low. The effect of high energy on high-frequency waves is that of dissipating the inward waves, since high-frequency waves are mainly outward propagating; as a result, outgoing waves are allowed to propagate almost undamped, and their energy content is therefore higher. Note that in the Kolmogorov case a dip, very close to the coronal base, appears as a signature of vanishing ingoing waves, a feature of the low-frequency reflected waves. This results in a vanishing absolute dissipation (heating), which is not found for the flat case and has important consequences for the acceleration and heating of the solar wind.

In the following we consider only a Kolmogorov spectrum. In Figure 9 the rms amplitude of the velocity field fluctuation integrated over the whole spectrum is plotted as a function of heliocentric distance (solid line; with the dotted line we also plot the magnetic field fluctuation in velocity units), along with some observational data (taken from Cranmer \& van Ballegooijen 2005), to which we address the following comments.

1. Filled diamonds show nonthermal line-broadening velocities measured by SUMER on the disk (Wilhelm et al. 1995).

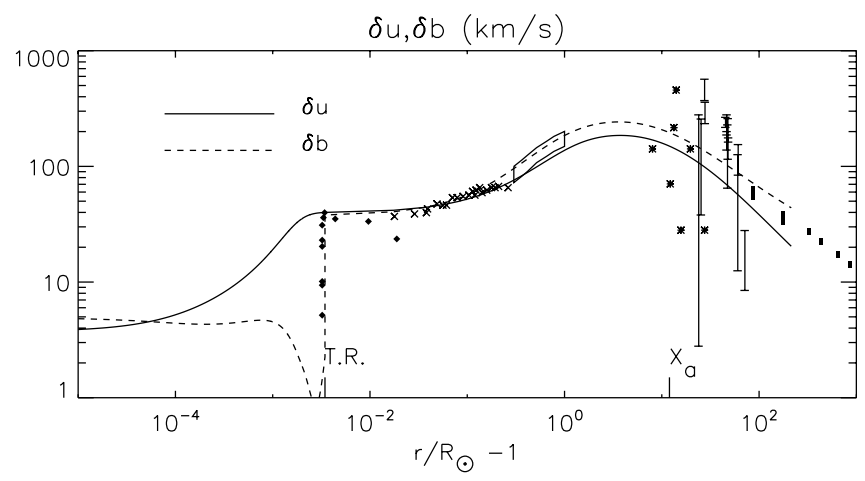

FIG. 9.- The rms amplitudes $\delta u$ and $\delta b$ (in velocity units) as functions of heliocentric distance for a photospheric Kolmogorov spectrum with $\delta u=40 \mathrm{~km} \mathrm{~s}^{-1}$ at the coronal base. Symbols indicate observational constraints (see text for explanation). 
2. Crosses show nonthermal velocities derived from SUMER observations above the solar limb (Banerjee et al. 1998).

3. The box represents the upper and lower limit given by Esser et al. (1999) from UVCS off-limb data.

4. Stars show early measurements from Armstrong \& Woo (1981).

5. The bars show recent measurements of transverse velocity field fluctuation using radio scintillation (Canals et al. 2002).

6. Filled bars show the Helios and Ulysses data for the Elsässer energies from Bavassano et al. (2000b), rewritten in terms of the velocity field fluctuation, assuming equipartition between magnetic and kinetic energy.

Note that the Helios and Ulysses data are obtained by averaging over periods $\gtrsim 1 \mathrm{hr}$ (corresponding to $\omega \lesssim 10^{-4} \mathrm{~Hz}$ ), while all the other points in the figure refers to rms values.

The overall agreement is quite good, even if data suggest a smaller power (more dissipation) just above the transition region and a larger power (less dissipation) at about $2 R_{\odot}$. Note that because of the equipartition assumption the Helios and Ulysses data disagree with the integrated quantities (the correct comparison has already been made above in Fig. 8). As noted by Cranmer \& van Ballegooijen (2005), the longitudinal velocity fluctuation data ( filled diamonds) agree very well with the magnetic field fluctuation amplitudes (dashed line) and could indicate wave coupling among the transverse and longitudinal modes. At leading order, compressive effects are driven by the magnetic pressure originating from the incompressible fluctuations and represent a way for Alfvén waves to get rid of the energy excess above the transition region. If these compressional waves are isotropic and suffer some dumping via shock formation, or other processes active in the low corona, they can reproduce the measured parallel $\delta u$ and supply the heating needed by the current model of wind acceleration.

\section{CONCLUSIONS}

In this paper we have modeled the nonlinear evolution of Alfvén waves propagating through the photosphere, the corona, and the solar wind up to $1 \mathrm{AU}$. Nonlinear interactions occur between outward-propagating and reflected waves, and it is assumed that a nonlinear cascade develops preferentially in a direction perpendicular to that of propagation, which we take to coincide with the direction of the mean radial magnetic field. While the phenomenological nonlinear term acts as a dissipative sink for both outward and inward waves independently of the wave frequency, reflection, provided by the stratification of the layer, is generally strong at low frequencies and decreases with increasing frequency.

We find that most of the heating occurs in the low corona (below the Alfvénic critical point), while very little power is dissipated below the transition region. For reasonable velocity field fluctuations at the base of the photosphere, a sufficient amount of energy flux is transmitted through the transition region. The adopted frequency coupling is not able to reproduce the observed spectral slope and evolution in the Alfvénic range even though frequency-integrated data at large distances constrain the outer spectrum to be steep ( $-5 / 3$ slope). The modification of the frequency spectrum occurs mainly in the chromosphere and in the photosphere, since waves experience a strong reflection at all the frequencies considered, while in the corona and the solar wind, the spectrum maintains approximately the same shape one finds at the coronal base.
Nonlinear dissipation based on reflection acts in different ways depending both on the (ingoing and outgoing) wave amplitude and on the layer considered. In the corona, reflection is not very high, but the outgoing wave amplitude is allowed to grow, so that the wave evolution is driven by the nonlinear interactions (all the modes evolve in the same way), and one finds a strong heating rate in the sub-Alfvénic corona. In the photosphere-chromosphere, a strong reflection rate, combined with small wave amplitudes, leads to an evolution similar to the linear case, which depends on frequency, and a small heating rate. As a result, most of the wave energy dissipation takes place in the first $4 R_{\odot}$ above the coronal base. The driving modes for dissipation are the modes that experience the biggest reflection, generally low-frequency modes. However, depending on the model of atmosphere, i.e., on its characteristic scale height, and on the energy distribution, i.e., flat or steep spectra, intermediate-frequency modes can be important as well.

The spectral shape varies mainly below the transition region; it steepens at low to intermediate frequencies $\left(10^{-5} \mathrm{~Hz} \lesssim \omega \lesssim\right.$ $\left.10^{-3} \mathrm{~Hz}\right)$ and flattens at high frequencies $\left(\omega \gtrsim 10^{-3} \mathrm{~Hz}\right)$, showing the characteristic features (energy peaks and frequency distribution) one finds in the transmission coefficient (linear behavior). In the corona it maintains approximately the shape one finds beyond the transition region because of the form of the nonlinear term adopted. The very low frequency range $\left(\omega \lesssim 10^{-5} \mathrm{~Hz}\right)$ practically does not evolve in the whole layer, and it keeps the original slope at the photosphere. With this model of nonlinearities, one can conclude that the spectrum one finds at $1 \mathrm{AU}$ is basically the same spectrum at the base of the corona. The input spectrum at the photosphere, whatever the shape is, is instead strongly modified by the transmission properties of the atmosphere below the transition region (independently of the model used for the nonlinear interaction). The energy peaks in the spectrum, resulting from an enhanced transmission at high frequencies, indicate that, even in the presence of nonlinear interactions, the photospheric layer acts as a filter for the energy injected through photospheric footpoint motion. If a smoothing of the forcing frequency is to be present, it must occur in this highly stratified layer. The data at large distances suggest that the energy at high frequency should be very low; however, we find an energy increase at high frequency. Since the spectral evolution in the corona also depends on the approximate frequency coupling contained in the nonlinear term, constraints on the photospheric input spectrum cannot be given safely. Given that high-frequency waves are transmitted through the transition region and are quite energetic in the very low corona, some other mechanism must be invoked to dissipate high-frequency waves, or a better modeling of the nonlinearities, which we plan to do in future works, is needed. As first pointed out by Hollweg (1981), such a high-frequency energy reservoir can be the source for plasma heating processes operating in the low corona. Note that not only do the peaks contribute to the energy budget, but the general flattening of the spectrum is important as well. A comparison with measurements of $\delta u$ suggests that the model can be considered a very good approximation in the outer corona and solar wind, while, despite the good agreement found in the low corona, some other processes must be invoked to reproduce the observed features below the Alfvénic critical point, such as compressible effects and wave coupling, especially in the chromosphere and photosphere. Other models of turbulent transport have been constructed to fit the decay of turbulence with distance from the Sun in the solar wind beyond $1 \mathrm{AU}$ (Smith et al. 2001; Breech et al. 2005), as well as to explain the extended heating in this region. Here the Alfvén speed can be neglected in the transport of the fluctuations, so that in some sense our model equations 
should be consistent with theirs, when rewritten in terms of the second-order moments. A generalization of turbulence transport equations consistent in the corona, acceleration region, and solar wind is a topic of current research.
We would like to thank the IPAM program "Grand Challenge Problems in Computational Astrophysics" at UCLA, where this work was completed. We also thank S. Oughton and W. H. Matthaeus for useful discussion.
Armstrong, J. W., \& Woo, R. 1981, A\&A, 103, 415

Banerjee, D., Teriaca, L., Doyle, J. G., \& Wilhelm, K. 1998, A\&A, 339, 208

Bavassano, B., Pietropaolo, E., \& Bruno, R. 2000a, J. Geophys. Res., 105, 12697

2000b, J. Geophys. Res., 105, 15959

Breech, B., Matthaeus, W. H., Minnie, J., Oughton, S., Parhi, S., Bieber, J. W., \& Bavassano, B. 2005, Geophys. Res. Lett., 32, 06103

Canals, A., Breen, A. R., Ofman, L., Moran, P. J., \& Fallows, R. A. 2002, Ann. Geophys., 20, 1265

Casalbuoni, S., Del Zanna, L., Habbal, S. R., \& Velli, M. 1999, J. Geophys. Res., 104, 9947

Cranmer, S. R., \& van Ballegooijen, A. A. 2005, ApJS, 156, 265

Del Zanna, L., Velli, M., \& Londrillo, P. 2001, A\&A, 367, 705

Dmitruk, P., \& Matthaeus, W. H. 2003, ApJ, 597, 1097

Dmitruk, P., Matthaeus, W. H., \& Lanzerotti, L. J. 2004, Geophys. Res. Lett., 31, 21805

Dmitruk, P., Matthaeus, W. H., Milano, L. J., \& Oughton, S. 2001a, Phys. Plasmas, 8, 2377

Dmitruk, P., Milano, L. J., \& Matthaeus, W. H. 2001b, ApJ, 548, 482

Dmitruk, P., et al. 2002, ApJ, 575, 571

Dobrowolny, M., Mangeney, A., \& Veltri, P. 1980, Phys. Rev. Lett., 45, 144

Esser, R., et al. 1999, ApJ, 510, L63

Habbal, S. R., Esser, R., Guhathakurta, M., \& Fisher, R. R. 1995, Geophys. Res. Lett., 22, 1465

Heinemann, M., \& Olbert, S. 1980, J. Geophys. Res., 85, 1311

Hollweg, J. V. 1978, Sol. Phys., 56, 305 . 1981, Sol. Phys., 70, 25

Hollweg, J. V., Jackson, S., \& Galloway, D. 1982, Sol. Phys., 75, 35

Kopp, R. A., \& Holzer, T. E. 1976, Sol. Phys., 49, 43

Krogulec, M., \& Musielak, Z. E. 1998, Acta Astron., 48, 77

Leroy, B. 1980, A\&A, 91, 136

Li, X., Habbal, S., Hollweg, J. V., \& Esser, R. 1999, J. Geophys. Res., 104, 2521

\section{EFERENCES}

Mangeney, A., Grappin, R., \& Velli, M. 1991, in Advances in Solar System Magnetohydrodynamics, ed. E. R. Priest \& A. W. Hood (Cambridge: Cambridge Univ. Press), 327

Matthaeus, W. H., Montgomery, D. C., \& Goldstein, M. L. 1983, Phys. Rev. Lett., 51, 1484

Matthaeus, W. H., Smith, C. W., \& Oughton, S. 1998, J. Geophys. Res., 103, 6495 Matthaeus, W. H., Zank, G. P., Oughton, S., Mullan, D. J., \& Dmitruk, P. 1999, ApJ, 523, L93

Matthaeus, W. H., Zhou, Y., Zank, G. P., \& Oughton, S. 1994, J. Geophys. Res., 99,23421

Munro, R. H., \& Jackson, B. V. 1977, ApJ, 213, 874

Oughton, S., Dmitruk, P., \& Matthaeus, W. H. 2004, Phys. Plasmas, 11, 2214

Oughton, S., et al. 2001, ApJ, 551, 565

Pruneti, F., \& Velli, M. 1997, in Fifth SOHO Workshop: The Corona and Solar Wind Near Minimum Activity, ed. A. Wilson (ESA SP-404; Noordwijk: ESA), 623

Shebalin, J. V., Matthaeus, W. H., \& Montgomery, D. 1983, J. Plasma Phys., 29, 525

Similon, P. L., \& Zargham, S. 1992, ApJ, 388, 644

Smith, C. W., Matthaeus, W. H., Zank, G. P., Ness, N. F., Oughton, S., \& Richardson, J. D. 2001, J. Geophys. Res., 106, 8253

Suzuki, T. K., \& Inutsuka, S.-i. 2005, ApJ, 632, L49

Tu, C.-Y. 1988, J. Geophys. Res., 93, 7

Tu, C.-Y., Pu, Z.-Y., \& Wei, F.-S. 1984, J. Geophys. Res., 89, 9695

Velli, M. 1993, A\&A, 270, 304

Velli, M., Grappin, R., \& Mangeney, A. 1989, Phys. Rev. Lett., 63, 1807

1991, Geophys. Astrophys. Fluid Dyn., 62, 101

Verdini, A., Velli, M., \& Oughton, S. 2005, A\&A, 444, 233

Wilhelm, K., et al. 1995, Sol. Phys., 162, 189

Zank, G. P., Matthaeus, W. H., \& Smith, C. W. 1996, J. Geophys. Res., 101, 17093

Zhou, Y., \& Matthaeus, W. H. 1989, Geophys. Res. Lett., 16, 755

1990, J. Geophys. Res., 95, 10291 